\title{
Unwind and slow down: checkpoint activation by helicase and polymerase uncoupling
}

\author{
David Cortez ${ }^{1}$ \\ Department of Biochemistry, Vanderbilt University, Nashville, Tennessee 37232, USA
}

Phosphoinositide 3-kinase related protein kinases (PIKK) including ataxia-telangiectasia mutated (ATM), DNAdependent protein kinase (DNA-PK), and ATM and Rad3-related (ATR) coordinate cellular responses to DNA damage. DNA-PK and ATM are primarily activated by double-strand breaks. The ATR kinase, in contrast, responds to numerous forms of genotoxic stress including intrastrand cross-links, oxidative damage, and polymerase toxins. At first glance, the disparate DNA structures that activate ATR impose a difficult biochemical challenge for damage sensing. However, recent data indicate that one DNA structure-single-stranded DNA (ssDNA) coated with a single-stranded DNA-binding protein (RPA) - is a common intermediate to activate ATR signaling in response to all of these genotoxic lesions. In this issue, Cimprich and colleagues (Byun et al. 2005) define how DNA damage and polymerase toxins use a common mechanism to generate the RPA-ssDNA needed to activate ATR. The method is the decoupling of helicase and polymerase activities at a replication fork.

\section{Linking RPA-coated ssDNA to checkpoint kinase activation}

The ATM and ATR protein kinases share many biochemical and functional similarities. Both are large protein kinases with significant sequence homology and a strong preference to phosphorylate serine or threonine residues followed by glutamine. Both target an overlapping set of substrates that promote cell cycle arrest. However, ATR is essential for the viability of replicating human and mouse cells while ATM is not (Brown and Baltimore 2000; Cortez et al. 2001). ATM functions in response to rare occurrences of double-strand breaks. ATR is activated during every $S$ phase to regulate the spatial and temporal firing of replication origins, the repair of damaged replication forks, and to prevent the pre-

${ }^{1}$ Correspondence.

E-MAIL david.cortez@vanderbilt.edu; FAX (615) 343-0704.

Article and publication are at http://www.genesdev.org/cgi/doi/10.1101/ gad.1316905. mature onset of mitosis (Nyberg et al. 2002; Shechter et al. 2004b). ATM mutations predispose carriers to develop cancer and are found in $\sim 1 \%$ of people. ATR mutations are rare and probably only compatible with viability when heterozygous or hypomorphic. While the only clear link between ATR mutation and disease is in a few patients with the rare Seckel syndrome (O'Driscoll et al. 2003), disruptions in the ATR pathway do cause genomic instability, and the ATR signaling pathway is activated by most cancer chemotherapies. Thus, the mechanistic details of how ATR functions are being actively investigated by numerous laboratories.

One clue to how ATR might differ from ATM came from the identification of a protein (ATRIP) that selectively binds to ATR (Cortez et al. 2001). ATRIP and ATR are almost always found in a complex, and the stability of each of these proteins is dependent on the other. ATRIP is essential for all known ATR activities and deletion of ATRIP phenocopies deletion of ATR. Recently, a C-terminal domain of ATRIP that binds an N-terminal domain of ATR was identified (Ball et al. 2005; Falck et al. 2005). Interestingly, there are similarities in a small part of the ATR-binding domain of ATRIP to regions in the Ku80 and Nbs1 proteins that bind to their respective PIKK partners DNA-PK and ATM (Falck et al. 2005). Ku and Nbs1 help to localize DNA-PK and ATM to doublestrand breaks (Dvir et al. 1992; Gottlieb and Jackson 1993; Carson et al. 2003; Kitagawa et al. 2004; Falck et al. 2005). Might ATRIP also serve as a recruitment module for the ATR kinase? Indeed, ATRIP is required for ATR localization to damage-induced nuclear foci (Ball et al. 2005), and ATRIP is required for ATR to bind DNA in Xenopus extracts (Kumagai et al. 2004).

A second important difference between ATR and ATM signaling should be appreciated. While ATM can be activated throughout the cell cycle, ATR activation is usually linked to ongoing DNA synthesis (Hekmat-Nejad et al. 2000; Lupardus et al. 2002; Stokes et al. 2002; Tercero et al. 2003). For example, in Xenopus extracts ATR associates with chromatin specifically during replication (Hekmat-Nejad et al. 2000) and inhibition of replication blocks ATR-dependent signaling (Lupardus et al. 2002; 
Stokes et al. 2002). In human cells, ATR activation after ultraviolet radiation (UV) also requires replication (Ward et al. 2004). This suggests that proteins or DNA structures that are specific to replicating cells may be needed to activate ATR. One possibility is that the replication fork itself is being sensed. A second possibility, and the one supported by the Cimprich data, is that a structure efficiently generated by replication enzymes (RPAssDNA) is sensed by ATR-ATRIP (Byun et al. 2005).

Several experiments indicate that RPA-ssDNA is essential for the activation of ATR checkpoint signaling. First, depletion of RPA from Xenopus extracts prevents ATR activation by topoisomerase inhibitors (Costanzo et al. 2003). Second, depletion of RPA from human cells with RNA interference prevents ATR-ATRIP localization to nuclear foci and phosphorylation of the ATR substrate Chk1 in response to UV radiation (Zou and Elledge 2003). Third, mutants in yeast RPA exhibit a checkpoint defect (Longhese et al. 1996; Kim and Brill 2001). Fourth, ATRIP can bind to RPA-ssDNA (Zou and Elledge 2003). Deletion of the RPA-ssDNA-binding domain on ATRIP prevents it from accumulating in nuclear foci that contain DNA lesions or stalled replication forks (Ball et al. 2005). Finally, RPA-ssDNA is required for the Rad17dependent clamp loader to load a damage-specific clamp containing the human Rad9, Rad1, and Hus1 (9-1-1) proteins (Ellison and Stillman 2003; Zou et al. 2003). The 9-1-1 complex is essential for ATR checkpoint signaling. Thus, RPA-ssDNA serves a similar purpose for ATR as a double-strand break serves for ATM.

\section{How to make RPA-ssDNA}

If RPA-ssDNA is the DNA structure that activates the ATR-ATRIP checkpoint kinase, then how is it generated by diverse genotoxic stresses? Two hypotheses have been advanced. First, RPA-ssDNA is an intermediate in many DNA repair processes. For example, the repair of a UV-induced thymine dimer by nucleotide excision repair proceeds through nuclease and helicase steps that expose a ssDNA gap containing RPA. A second hypothesis is that the stalling of a DNA polymerase without the concomitant stalling of the DNA helicase could generate regions of RPA-ssDNA at a replication fork. Polymerase inhibitors, such as aphidicolin, activate ATR and cause extensive unwinding of plasmid DNA placed in a replication competent Xenopus egg extract (Walter and Newport 2000). DNA lesions that block DNA polymerases do not interfere with the viral T-antigen helicase in the SV40 replication system (Cordeiro-Stone et al. 1999; Veaute et al. 2000). Is the eukaryotic replicative helicase also insensitive to DNA lesions? Cimprich and colleagues (Byun et al. 2005) now demonstrate conclusively that functional uncoupling of helicase and polymerase activities does occur and is required for checkpoint signaling in Xenopus extracts.

They begin by demonstrating that UV or cisplatindamaged plasmids (containing intrastrand cross-links) are extensively unwound in replication-competent extracts. Importantly, unwinding is correlated with Chk1 phosphorylation. Chk1 is directly phosphorylated and activated by ATR and is required for cell cycle arrest (Liu et al. 2000). The timing of Chk1 phosphorylation was slightly delayed compared with ssDNA formation, and as replication converted the unwound plasmid to duplex DNA, Chk1 phosphorylation disappeared. The authors then demonstrate that unwinding of the plasmid is dependent on the action of the MCM helicase. A fragment of the $\mathrm{Rb}$ protein that binds MCM7 and inhibits helicase activity (Sterner et al. 1998) blocks unwinding, as do neutralizing antibodies to CDC45, which functions as a cofactor for the MCM complex (Pacek and Walter 2004). Most importantly, when unwinding was inhibited, ATR no longer phosphorylated Chk1. Thus, the presence of the damaged DNA by itself is insufficient to generate a checkpoint signal. Instead, an interaction between the lesion and a replication fork is needed (Fig. 1).

These data indicate that the difference in the ability of the polymerase and helicase to use a damaged template

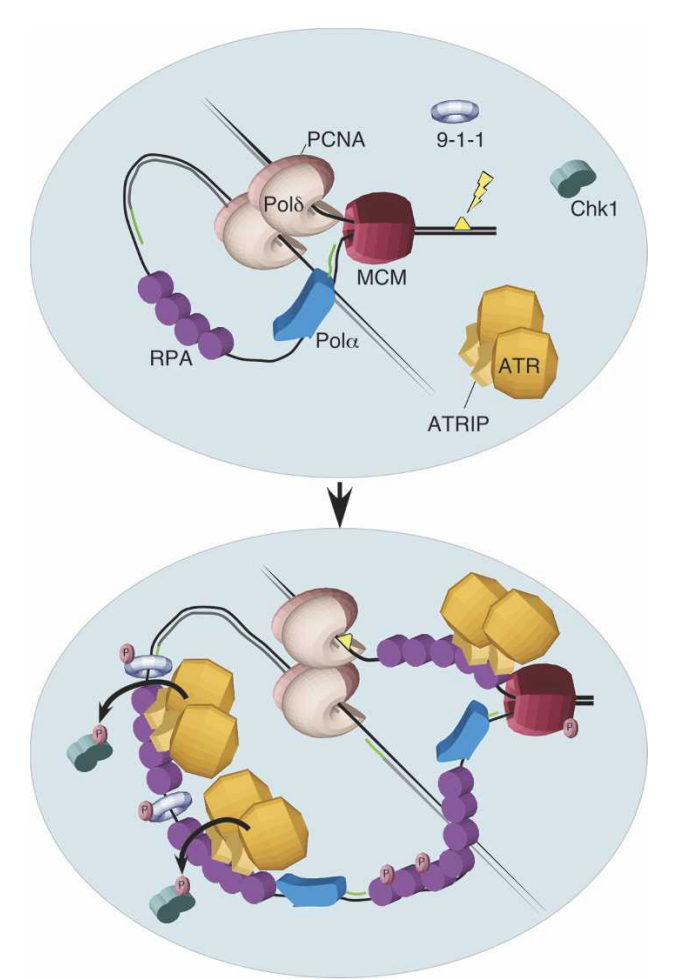

Figure 1. A simplified model for how decoupling of the replicative helicase and polymerase generates RPA-ssDNA and promotes ATR activation. A DNA lesion does not block the MCM helicase from unwinding the DNA duplex, but it does stall the DNA polymerase. RPA binds to the single-stranded DNA that accumulates, and RPA-ssDNA promotes loading of the 9-1-1 and ATR-ATRIP complexes. The juxtaposition of these complexes allows ATR to phosphorylate Chk1, which then promotes cell cycle arrest. Other proteins at the replication fork are also phosphorylated by ATR, including the 9-1-1 complex, RPA, and the MCM complex. Many additional checkpoint proteins including Claspin, TopBP1/Cut5, and the Rad17 clamp loading complex are present at the stalled replication fork and promote Chk1 phosphorylation but are not placed in this diagram. 
provides the mechanism of generating the RPA-ssDNA required to activate the checkpoint. One implication of these results is that lesions that prevent the helicase from unwinding may not elicit an ATR-dependent checkpoint response. It would be interesting to determine whether an interstrand cross-link would block the helicase and/or elicit a checkpoint response in this system. Interstrand cross-linking agents such as mitomycin C, nitrogen mustard, and photoactivated psoralen do elicit ATR signaling when added to cultured cells (Lambert et al. 2003; Pichierri and Rosselli 2004); however, these agents also cause intrastrand cross-links, and their repair can proceed through an intrastrand cross-link intermediate, thus, it is unclear what is actually being sensed by ATR. It will also be important to examine how the specific placement of an intrastrand lesion on either the leading or lagging strand causes differences in the checkpoint response. Lagging-strand lesions may cause only small gaps of ssDNA instead of extensive unwinding because Okazaki fragment synthesis can reinitiate downstream of the lesion (Meneghini and Hanawalt 1976; Svoboda and Vos 1995). Unfortunately, since plasmids in Xenopus extracts initiate replication randomly, other systems will be needed to answer this question.

Another conclusion of the paper is that the amount of RPA-ssDNA generated determines the extent of Chk1 activation. Previous experiments in yeast had suggested that the number of active replication forks may determine a threshold required for ATR activation (Shimada et al. 2002). By performing careful molar and mass equivalent experiments with different size plasmids, Cimprich and colleagues (Byun et al. 2005) determined that the extent to which ssDNA is produced rather than the number of forks determines the amount of ATR activation in Xenopus extracts. It remains possible that there is a threshold to the ATR response, but perhaps in animal cells the threshold is reflected in different ATR signal strengths needed to activate different types of local or cell-wide checkpoint responses instead of a threshold for ATR activation per se. If one considers RPAssDNA as a ligand for a checkpoint receptor (analogous to a growth factor-receptor complex), then it is not surprising that there should be a dose-responsive activation of the checkpoint signal. The biochemical basis of this response may be the amount of ATR-ATRIP and/or 9-1-1 loaded onto the RPA-ssDNA.

\section{Multiple receptors for the ssDNA-RPA ligand}

If RPA-ssDNA is a ligand that activates ATR-dependent signaling, then what is the receptor for this ligand? ATRIP seems to be the likely candidate. It contains an $\mathrm{N}$-terminal domain that is necessary and sufficient to bind to RPA-ssDNA, which is separable from its C-terminal ATR-binding domain (Ball et al. 2005). ATRIP binding to RPA-ssDNA promotes ATR association with DNA in vitro (Zou and Elledge 2003). ATRIP is also essential for ATR to localize to nuclear foci after DNA damage in vivo. Mutations in its N-terminal RPA-binding domain severely impair relocalization of ATRIP to
DNA-damage-induced foci (Ball et al. 2005). Thus, ATRIP would appear to be at least one of the receptors for the RPA-ssDNA ligand.

However, recently published data indicate that a direct ATRIP-RPA-ssDNA interaction is neither sufficient for ATR-ATRIP localization to sites of DNA damage nor essential for ATR activation. ATR is not retained at or activated by stalled replication forks in Nbs1-deficient cells despite RPA-ssDNA accumulation (Stiff et al. 2005). ATR is also not retained at UV-induced lesions in H2AX-deficient cells, but it is activated and phosphorylates Chk1 in these cells (Ward et al. 2004). There are RPA-ssDNA-independent means of ATR-ATRIP association with DNA as well (Unsal-Kacmaz et al. 2002; Bomgarden et al. 2004; Peng and Chen 2005). Furthermore, while deletion of the $\mathrm{N}$ terminus of ATRIP prevents RPA-ssDNA binding and accumulation of ATRIP at sites of DNA damage, the mutant ATRIP protein still supports ATR-dependent Chk1 phosphorylation in cells (Ball et al. 2005). This result suggests that accumulation of the ATR-ATRIP complex in nuclear foci is separable from ATR activation. It should be noted that accumulation into immunofluorescently distinct foci is not a sensitive assay for recognition of DNA damage. Perhaps ATR-ATRIP can be recruited to the uncoupled replication fork without ATRIP binding to RPA-ssDNA, but the ATRIP-RPA-ssDNA interaction promotes retention and accumulation of ATR. These results should also be considered in relation to the observation that ATM activation is separable from its retention at double-strand breaks (Kitagawa et al. 2004; Falck et al. 2005). Similarly, there may be multiple steps to ATR activation, and the phosphorylation of different substrates probably requires different biochemical interactions.

There may be multiple receptors for RPA-ssDNA that participate in the checkpoint response. Cut5 (TOPBP1) participates in the loading of ATR onto RPA-ssDNA, and Cut5 loading is dependent on RPA after DNA damage (Parrilla-Castellar and Karnitz 2003). 9-1-1 complex loading by Rad17 also requires RPA-ssDNA but is independent of ATR-ATRIP (Ellison and Stillman 2003; Zou et al. 2003). Furthermore, RPA mutants that abolish interactions between RPA and replication factor C (RFC) in vitro cause checkpoint deficiencies in vivo (Kim and Brill 2001). Thus, there may be multiple receptors for the RPA-ssDNA ligand, and the original problem of how diverse DNA structures could activate a common signaling molecule seems to have been turned on its head.

\section{A second signal for checkpoint activation}

While RPA-ssDNA is essential for ATR signaling, it is not sufficient. Indeed, RPA-ssDNA by itself does not activate ATR in Xenopus extracts (Stokes et al. 2002). The Rad17-dependent loading of the 9-1-1 trimer at sites of DNA damage is essential for ATR to phosphorylate at least some of its substrates including Chk1 (Zou et al. 2002). In vitro, RPA-ssDNA and a recessed DNA strand end are required to stimulate 9-1-1 loading (Ellison and Stillman 2003; Zou et al. 2003). There are conflicting 
reports as to what type of end $\left(3^{\prime}\right.$ or $\left.5^{\prime}\right)$ is preferred by the Rad17 clamp loader. A recessed 3 '-end generated by Pol $\alpha$ (a primer/template junction) is what is normally recognized by the RFC complex to load PCNA, whereas a recessed 5 '-end would need to be generated by other enzymatic processing.

Cimprich and colleagues (Byun et al. 2005) also find that a second signal in addition to RPA-ssDNA is required for ATR to phosphorylate Chk1. High concentrations of aphidicolin or neutralizing antibodies to Pol $\alpha$ do not block unwinding of a damaged plasmid but do inhibit Chk1 phosphorylation. This is only true if the inhibitors are added prior to initiation of replication. Since these agents block the DNA synthesis but not RNA primase function of Pol $\alpha$, extension of the RNA primer must be required for Chk1 phosphorylation. Importantly, ATR-ATRIP, RPA, and Pol $\alpha$ are still chromatinassociated under these circumstances, but the 9-1-1 complex is not. Thus, the second, Pol $\alpha$-dependent signal promotes 9-1-1 loading.

The exact structure formed by Pol $\alpha$ that activates the checkpoint is still unclear. If the 9-1-1 complex is loaded at a recessed 3 '-end in vivo, then perhaps all that is required is the DNA extension at the primer-template junction. However, since a primed ssDNA template treated with MMS is insufficient to activate the checkpoint (Stokes et al. 2002), and recessed $5^{\prime}$-ends may be more efficient for 9-1-1 loading than recessed 3 '-ends (Ellison and Stillman 2003), something more may be required. In addition, the amount of Pol $\alpha$ on chromatin increases in extracts treated with aphidicolin; therefore, multiple extended primers may be synthesized on the lagging strand, thereby providing a means to amplify the checkpoint signal (Michael et al. 2000).

Another question is why has the checkpoint evolved to require these two apparently independent events (ATR-ATRIP and 9-1-1 recruitment). Perhaps this twostep mechanism ensures that only ssDNA generated during specific types of activities can lead to Chk1-dependent cell cycle arrest. Yeast ATR is activated (as measured by yeast ATRIP phosphorylation) after DNA damage even in 9-1-1 mutants (Edwards et al. 1999). Are there other ATR substrates that do not require 9-1-1 loading? Mechanistically, why is 9-1-1 loading required for Chk1 phosphorylation? Does it recruit substrates to ATR? Structurally, the 9-1-1 complex resembles the PCNA clamp, so what is it clamping? Many additional checkpoint proteins including Claspin are also required for Chk1 phosphorylation. Thus, further research is required for a full mechanistic understanding of ATR signaling.

\section{Other roles of the MCM complex in checkpoint signaling}

One of the surprising results of the Cimprich study (Byun et al. 2005) is that an entire 10-kb plasmid is unwound by the MCM helicase after the polymerase is stalled. Replication of this size plasmid initiates from a single site in Xenopus extracts. Only an extra 100-200 bp of ssDNA are formed at a stalled replication fork after checkpoint activation in wild-type yeast cells (Sogo et al. 2002). Checkpoint deficiencies cause the length of the ssDNA tract to increase to between 500 and $800 \mathrm{bp}$. This suggests that one activity of the ATR checkpoint may be to inhibit ssDNA formation. The mammalian and Xenopus MCM complexes are phosphorylated by ATR (Cortez et al. 2004; Yoo et al. 2004). The function of the phosphorylation is unclear, but it is tempting to speculate that it could slow helicase activity to prevent the generation of long ssDNA tracts. Why then do the damaged plasmids in the Xenopus extracts completely unwind? Is this unique to this in vitro system or is the amount of ssDNA tolerated in animal cells larger than that in yeast?

In addition to being a substrate of ATR, the MCM complex also can physically bind to ATRIP and Rad17 (Cortez et al. 2004; Tsao et al. 2004). A C-terminal region of MCM7 mediates these interactions, which intriguingly is also the interaction domain for the $\mathrm{Rb}$ fragment that the Cimprich group (Byun et al. 2005) used to inhibit MCM activity. Partial depletion of MCM7 by RNA inhibition impairs ATR signaling, whereas depletion of other MCM subunits does not have this effect (Cortez et al. 2004; Tsao et al. 2004). In these experiments there is no detectable decrease in the rate of replication at the levels of MCM depletion achieved. While it is difficult to rule out that depletion of MCM7 impaired unwinding activity of the entire helicase complex in these experiments, the biochemical connection between MCM7, ATRIP, and Rad17 indicates that MCM7 may have a more direct role in checkpoint signaling.

\section{Conclusions}

The Cimprich data provide clear evidence that decoupling of helicase and polymerase activities to generate RPA-ssDNA is necessary but not sufficient for DNA damage and polymerase inhibitors to activate ATR in Xenopus extracts (Byun et al. 2005). It seems unlikely that this will be the only means of activating ATR. ssDNA gaps may activate ATR outside of $S$ phase but probably rarely persist because of rapid repair activities (Costanzo and Gautier 2003; Stiff et al. 2005). ATR may also have some activity even at normal replication forks to regulate origin firing (Marheineke and Hyrien 2004; Shechter et al. 2004a). Nonetheless, Cimprich and colleagues (Byun et al. 2005) have defined how decoupling of helicase and polymerase function through intrinsic differences in their substrate compatibilities provides a method of promoting checkpoint signaling in response to diverse genotoxins.

\section{Acknowledgments}

I thank members of the Cortez laboratory for their helpful discussions. Research in the author's laboratory is supported by grants from the NIH (K01CA93701 and R01CA102729) and the Pew Scholars program of the Pew Charitable Trusts. 


\section{References}

Ball, H.L., Myers, J.S., and Cortez, D. 2005. ATRIP binding to RPA-ssDNA promotes ATR-ATRIP localization but is dispensable for Chk1 phosphorylation. Mol. Biol. Cell 16: Epub ahead of print.

Bomgarden, R.D., Yean, D., Yee, M.C., and Cimprich, K.A. 2004. A novel protein activity mediates DNA binding of an ATR-ATRIP complex. J. Biol. Chem. 279: 13346-13353.

Brown, E.J. and Baltimore, D. 2000. ATR disruption leads to chromosomal fragmentation and early embryonic lethality. Genes \& Dev. 14: 397-402.

Byun, T.S., Pacek, M., Yee, M.-C., Walter, J.C., and Cimprich, K.A. 2005. Functional uncoupling of MCM helicase and DNA polymerase activities activates the ATR-dependent checkpoint Genes \& Dev. (this issue).

Carson, C.T., Schwartz, R.A., Stracker, T.H., Lilley, C.E., Lee, D.V., and Weitzman, M.D. 2003. The Mre11 complex is required for ATM activation and the G2/M checkpoint. EMBO J. 22: 6610-6620.

Cordeiro-Stone, M., Makhov, A.M., Zaritskaya, L.S., and Griffith, J.D. 1999. Analysis of DNA replication forks encountering a pyrimidine dimer in the template to the leading strand. J. Mol. Biol. 289: 1207-1218.

Cortez, D., Guntuku, S., Qin, J., and Elledge, S.J. 2001. ATR and ATRIP: Partners in checkpoint signaling. Science 294: 17131716.

Cortez, D., Glick, G., and Elledge, S.J. 2004. Minichromosome maintenance proteins are direct targets of the ATM and ATR checkpoint kinases. Proc. Natl. Acad. Sci. 101: 1007810083.

Costanzo, V. and Gautier, J. 2003. Single-strand DNA gaps trigger an ATR- and Cdc7-dependent checkpoint. Cell Cycle 2: 17 .

Costanzo, V., Shechter, D., Lupardus, P.J., Cimprich, K.A., Gottesman, M., and Gautier, J. 2003. An ATR- and Cdc7dependent DNA damage checkpoint that inhibits initiation of DNA replication. Mol. Cell 11: 203-213.

Dvir, A., Peterson, S.R., Knuth, M.W., Lu, H., and Dynan, W.S. 1992. Ku autoantigen is the regulatory component of a template-associated protein kinase that phosphorylates RNA polymerase II. Proc. Nat1. Acad. Sci. 89: 11920-11924.

Edwards, R.J., Bentley, N.J., and Carr, A.M. 1999. A Rad3-Rad26 complex responds to DNA damage independently of other checkpoint proteins. Nat. Cell Biol. 1: 393-398.

Ellison, V. and Stillman, B. 2003. Biochemical characterization of DNA damage checkpoint complexes: Clamp loader and clamp complexes with specificity for 5 ' recessed DNA. PLOS Biol. 1: E33.

Falck, J., Coates, J., and Jackson, S.P. 2005. Conserved modes of recruitment of ATM, ATR and DNA-PKcs to sites of DNA damage. Nature 434: 605-611.

Gottlieb, T.M. and Jackson, S.P. 1993. The DNA-dependent protein kinase: Requirement for DNA ends and association with $\mathrm{Ku}$ antigen. Cell 72: 131-142.

Hekmat-Nejad, M., You, Z., Yee, M.C., Newport, J.W., and Cimprich, K.A. 2000. Xenopus ATR is a replication-dependent chromatin-binding protein required for the DNA replication checkpoint. Curr. Biol. 10: 1565-1573.

Kim, H.S. and Brill, S.J. 2001. Rfc4 interacts with Rpal and is required for both DNA replication and DNA damage checkpoints in Saccharomyces cerevisiae. Mol. Cell. Biol. 21: 3725-3737.

Kitagawa, R., Bakkenist, C.J., McKinnon, P.J., and Kastan, M.B. 2004. Phosphorylation of SMC1 is a critical downstream event in the ATM-NBS1-BRCA1 pathway. Genes \& Dev.
18: $1423-1438$.

Kumagai, A., Kim, S.M., and Dunphy, W.G. 2004. Claspin and the activated form of ATR-ATRIP collaborate in the activation of Chk1. J. Biol. Chem. 279: 49599-49608.

Lambert, S., Mason, S.J., Barber, L.J., Hartley, J.A., Pearce, J.A., Carr, A.M., and McHugh, P.J. 2003. Schizosaccharomyces pombe checkpoint response to DNA interstrand cross-links. Mol. Cell. Biol. 23: 4728-4737.

Liu, Q., Guntuku, S., Cui, X.S., Matsuoka, S., Cortez, D., Tamai, K., Luo, G., Carattini-Rivera, S., DeMayo, F., Bradley, A. et al. 2000. Chk1 is an essential kinase that is regulated by Atr and required for the G(2)/M DNA damage checkpoint. Genes \& Dev. 14: 1448-1459.

Longhese, M.P., Neecke, H., Paciotti, V., Lucchini, G., and Plevani, P. 1996. The $70 \mathrm{kDa}$ subunit of replication protein $A$ is required for the G1/S and intra-S DNA damage checkpoints in budding yeast. Nucleic Acids Res. 24: 3533-3537.

Lupardus, P.J., Byun, T., Yee, M.C., Hekmat-Nejad, M., and Cimprich, K.A. 2002. A requirement for replication in activation of the ATR-dependent DNA damage checkpoint. Genes \& Dev. 16: 2327-2332.

Marheineke, K. and Hyrien, O. 2004. Control of replication origin density and firing time in Xenopus egg extracts: Role of a caffeine-sensitive, ATR-dependent checkpoint. I. Biol. Chem. 279: 28071-28081.

Meneghini, R. and Hanawalt, P. 1976. T4-endonuclease V-sensitive sites in DNA from ultraviolet-irradiated human cells. Biochim. Biophys. Acta 425: 428-437.

Michael, W.M., Ott, R., Fanning, E., and Newport, J. 2000. Activation of the DNA replication checkpoint through RNA synthesis by primase. Science 289: 2133-2137.

Nyberg, K.A., Michelson, R.J., Putnam, C.W., and Weinert, T.A. 2002. Toward maintaining the genome: DNA damage and replication checkpoints. Annu. Rev. Genet. 36: 617-656.

O'Driscoll, M., Ruiz-Perez, V.L., Woods, C.G., Jeggo, P.A., and Goodship, J.A. 2003. A splicing mutation affecting expression of ataxia-telangiectasia and Rad3-related protein (ATR) results in Seckel syndrome. Nat. Genet. 33: 497-501.

Pacek, M. and Walter, J.C. 2004. A requirement for MCM7 and Cdc45 in chromosome unwinding during eukaryotic DNA replication. EMBO J. 23: 3667-3676.

Parrilla-Castellar, E.R. and Karnitz, L.M. 2003. Cut5 is required for the binding of ATR and DNA polymerase $\alpha$ to genotoxindamaged chromatin. J. Biol. Chem. 278: 45507-45511.

Peng, A. and Chen, P.L. 2005. NFBD1/Mdc1 mediates ATRdependent DNA damage response. Cancer Res. 65: 11581163.

Pichierri, P. and Rosselli, F. 2004. The DNA crosslink-induced S-phase checkpoint depends on ATR-CHK1 and ATRNBS1-FANCD2 pathways. EMBO J. 23: 1178-1187.

Shechter, D., Costanzo, V., and Gautier, J. 2004a. ATR and ATM regulate the timing of DNA replication origin firing. Nat. Cell Biol. 6: 648-655.

- 2004b. Regulation of DNA replication by ATR: Signaling in response to DNA intermediates. DNA Repair (Amst) 3: 901-908.

Shimada, K., Pasero, P., and Gasser, S.M. 2002. ORC and the intra-S-phase checkpoint: A threshold regulates Rad53p activation in S phase. Genes \& Dev. 16: 3236-3252.

Sogo, J.M., Lopes, M., and Foiani, M. 2002. Fork reversal and ssDNA accumulation at stalled replication forks owing to checkpoint defects. Science 297: 599-602.

Sterner, J.M., Dew-Knight, S., Musahl, C., Kornbluth, S., and Horowitz, J.M. 1998. Negative regulation of DNA replication by the retinoblastoma protein is mediated by its association with MCM7. Mol. Cell. Biol. 18: 2748-2757. 
Stiff, T., Reis, C., Alderton, G.K., Woodbine, L., O'Driscoll, M., and Jeggo, P.A. 2005. Nbs1 is required for ATR-dependent phosphorylation events. EMBO J. 24: 199-208.

Stokes, M.P., Van Hatten, R., Lindsay, H.D., and Michael, W.M. 2002. DNA replication is required for the checkpoint response to damaged DNA in Xenopus egg extracts. I. Cell Biol. 158: 863-872.

Svoboda, D.L. and Vos, J.M. 1995. Differential replication of a single, UV-induced lesion in the leading or lagging strand by a human cell extract: Fork uncoupling or gap formation. Proc. Nat1. Acad. Sci. 92: 11975-11979.

Tercero, J.A., Longhese, M.P., and Diffley, J.F. 2003. A central role for DNA replication forks in checkpoint activation and response. Mol. Cell 11: 1323-1336.

Tsao, C.C., Geisen, C., and Abraham, R.T. 2004. Interaction between human MCM7 and Rad17 proteins is required for replication checkpoint signaling. EMBO J. 23: 4660-4669.

Unsal-Kacmaz, K., Makhov, A.M., Griffith, J.D., and Sancar, A. 2002. Preferential binding of ATR protein to UV-damaged DNA. Proc. Nat1. Acad. Sci. 99: 6673-6678.

Veaute, X., Mari-Giglia, G., Lawrence, C.W., and Sarasin, A. 2000. UV lesions located on the leading strand inhibit DNA replication but do not inhibit SV40 T-antigen helicase activity. Mutat. Res. 459: 19-28.

Walter, J. and Newport, J. 2000. Initiation of eukaryotic DNA replication: Origin unwinding and sequential chromatin association of Cdc45, RPA, and DNA polymerase alpha. Mol. Cell 5: 617-627.

Ward, I.M., Minn, K., and Chen, J. 2004. UV-induced ataxiatelangiectasia-mutated and Rad3-related (ATR) activation requires replication stress. J. Biol. Chem. 279: 9677-9680.

Yoo, H.Y., Shevchenko, A., and Dunphy, W.G. 2004. Mcm2 is a direct substrate of ATM and ATR during DNA damage and DNA replication checkpoint responses. I. Biol. Chem. 279: 53353-53364.

Zou, L. and Elledge, S.J. 2003. Sensing DNA damage through ATRIP recognition of RPA-ssDNA complexes. Science 300: 1542-1548.

Zou, L., Cortez, D., and Elledge, S.J. 2002. Regulation of ATR substrate selection by Rad17-dependent loading of Rad9 complexes onto chromatin. Genes \& Dev. 16: 198-208.

Zou, L., Liu, D., and Elledge, S.J. 2003. Replication protein Amediated recruitment and activation of Rad17 complexes. Proc. Natl. Acad. Sci. 100: 13827-13832. 


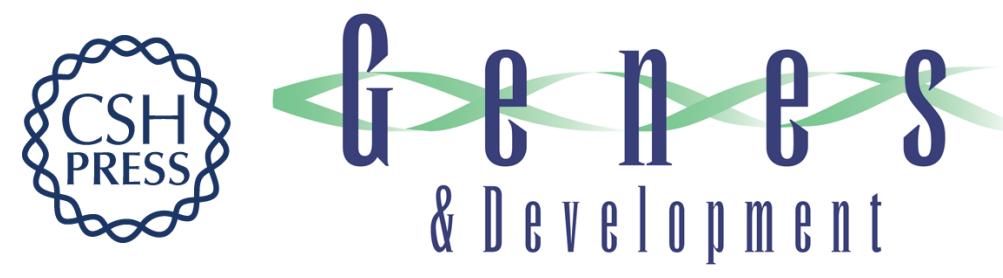

\title{
Unwind and slow down: checkpoint activation by helicase and polymerase uncoupling
}

\author{
David Cortez
}

Genes Dev. 2005, 19:

Access the most recent version at doi:10.1101/gad.1316905

$\begin{array}{ll}\text { References } & \begin{array}{l}\text { This article cites } 47 \text { articles, } 29 \text { of which can be accessed free at: } \\ \text { http://genesdev.cshlp.org/content/19/9/1007.full.html\#ref-list-1 }\end{array}\end{array}$

License

Email Alerting Receive free email alerts when new articles cite this article - sign up in the box at the top Service right corner of the article or click here.

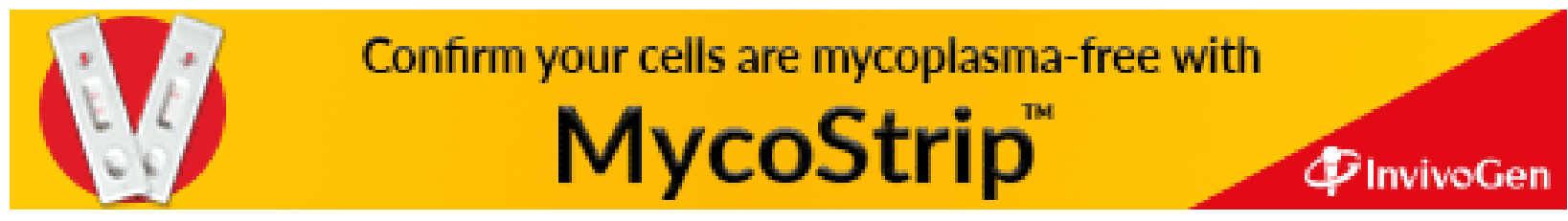

